\title{
Distributed Storage Performance Measurement IN A VIRTUALIZED ENVIRONMENT
}

\author{
Vedran Dakic, Davor Baksa \& Jasmin Rezepagic
}
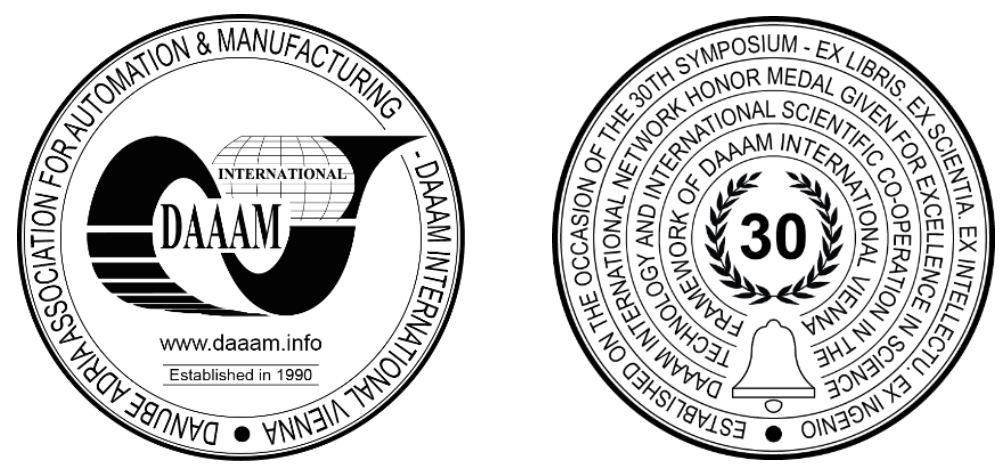

This Publication has to be referred as: Dakic, V[edran]; Baksa, D[avor] \& Redzepagic, J[asmin] (2021). Distributed Storage Performance Measurement in a Virtualized Environment, Proceedings of the 32nd DAAAM International Symposium, pp.0084-0089, B. Katalinic (Ed.), Published by DAAAM International, ISBN 978-3-902734-33-4, ISSN 1726-9679, Vienna, Austria

DOI: $10.2507 / 32$ nd.daaam.proceedings.012

\begin{abstract}
This paper describes a very simple, yet efficient methodology to measure storage performance in a virtualized environment. By "virtualized environment", we are talking about an environment that's based on a hypervisor and virtual machines, commonly used compute objects in IT industry today. Having in mind that virtual machines need a significant amount of storage in terms of capacity, lowest latency possible, and best possible throughput in terms of outright performance, having a capability to measure storage performance in a distributed environment is a welcome addition to any datacenter. If nothing else, it could give us a good performance baseline that we can use for future storage performance tuning. That's why - as a result of this paper - we want to use a virtual machine-based methodology to implement a BASH (Bourne Again SHell) shell script-based set of automated test procedures that are easy to use and don't require a lot of time to be interpreted.
\end{abstract}

Keywords: hypervisor; virtual machine; storage performance; open-source; multipathing

\section{Introduction}

Measuring storage performance in virtualized environments - our first goal - has always been a difficult issue to tackle. There are different problems in the way - which tests to use, how to simulate workloads, when to start workloads, which workloads to use... All of that is very important to the second subject of this paper, which is all about functionality and performance. The last step - which is fundamental to this paper - is actually about tuning storage and hypervisor settings to achieve better storage performance for that environment. The caveat being that every environment is different - different workloads, different servers, different switches, different cabling... The aim of this paper is primarily to introduce a testing methodology for storage performance measurement. After that, it's also about applying that methodology to a specific environment in all directions - top-down, bottom-up, left-right.

As a basis for this paper will use OSNEXUS Quantastor open-source storage system through various connectivity scenarios and across multiple platforms to determine whether such a system is suitable for use in a variety of business environments. Companies often require dedicated storage systems that they use in conjunction with virtualization technology, and the paper will try to simulate loads when using such systems and measure performance metrics with keeping track of all of the results, possible difficulties or benefits, conclude on the cost-effectiveness of such a system, and tuning specific hardware or software adapters and attributes with respect to the measured results. 


\section{Testing environment description}

Our testing environment aims to simulate a virtualized environment that uses some of the most commonly used solutions within the context of virtual machines and the performance of used disk systems. For this purpose, two HP Proliant DL380p Gen8 servers were prepared, one as a compute node, the other for storage. Server1 (compute node) took on the role of virtual machine hypervisor (VMware ESXi, a well-known hypervisor solution), while OSNEXUS system was installed on Server2 (storage system), which enables all capabilities of a SDS (Software Defined Storage) platform.

For testing purposes, we used Linux virtual machines, configured by using VMware paravirtual storage controller. VMware presents the Paravirtual virtual storage controller as the one to use when we need lower latency, better throughput, and lower CPU overhead from the host perspective [1]. In the Linux variant, sixteen (16) virtual machines based on the CentOS operating system were prepared, and LAMP (Linux-Apache-MySQL-PHP, Hypertext Preprocessor) was installed on each instance. Each virtual machine had a PHP-based website that uses the MySQL database and running on the Apache 2.4 platform. These virtual machines were using DD (Copy and Convert), IOPING, and FIO (Flexible I/O tester) tools to provide metrics such as bandwidth, latency, and IOPS for each environment used.

As for the storage system (Server2), 8 SSD (Solid State Disk) SATA (Serial Advanced Technology Attachment) disks and 1 NVMe (Non-Volatile Memory express) disk were used in the most commonly used RAID (Redundant Array of Inexpensive Disks) array configurations (RAID 5 and RAID 10 for SATA disks) and direct access for the NVMe disk. NVMe SSDs, as PCIe (PCI Express) devices, employ the high-speed PCIe bus to communicate with the host [2]. Also, NVMe is designed from the ground up for non-volatile memory technology such as NAND and 3D XPoint over PCIe [3]. Reasons behind our choices of SSDs and NVMe devices are:

- RAID 5 is often used in systems where attempts are made to ensure maximum capacity with guaranteed minimum redundancy (single drive redundancy - in a field with 8 disks, the usable capacity is 7). In this variant, more focus is added to capacity versus speed and redundancy.

- RAID 10 is also a commonly used RAID system configuration - especially in virtualized environments - where 50\% utilization of available disk capacity is ensured, but $50 \%$ of disk space is redundant and better performance versus RAID 5 array is ensured.

Both variants use $8 \mathrm{~K}$ and $64 \mathrm{~K}$ block sizes on a disk system presented to virtual machines to measure performance metrics depending on the purpose. For example, the $64 \mathrm{~K}$ variant is recommended for the general storage scenario while the $8 \mathrm{~K}$ variant is recommended for usage scenarios where the IOPS (Input/output Operations Per Second) metric versus raw bandwidth is more important - a good example for this usage model would be databases.

Also, two different connections of Server1 and Server2 were used - Ethernet and Fibre Channel (FC), in single-uplink (10 Gbit/s Ethernet or $8 \mathrm{Gbit} / \mathrm{s} \mathrm{FC)} \mathrm{and} \mathrm{four} \mathrm{links} \mathrm{using} \mathrm{multipathing} \mathrm{(40} \mathrm{Gbit/s} \mathrm{Ethernet} \mathrm{or} 32 \mathrm{Gbit} / \mathrm{s}$ FC) configurations to be able to measure scalability. For each iteration of the test, we also changed the amount of available working memory on the storage server ( $32 \mathrm{~GB} / 64 \mathrm{~GB}$ ) since OSNEXUS is designed to use the available working memory for the cache where possible. We can also tune the amount of memory that goes to caching out of the overall server memory amount, as it's one of the available options.

IOPS usage in idle state was tested for 24 hours, so we set up a network connection to a Synology NAS, installed Zabbix in a Docker container on Synology NAS (Network Attached Storage), and Zabbix agent on all of the VMs (Virtual Machines) to set everything up for IOPS monitoring. We initially started with running all of the tests manually (FIO, DD, IOPING) and typed in the table, but as we soon realized it didn't make sense because the manual process was slowing us down significantly. That's the reason why we created a script to do the work for us, as we will describe in "Testing methodology and storage performance measurement complexity".

While developing our script, we started with one virtual machine, tested everything, added a couple of virtual machines more, re-tested and, as the number of virtual machines grew, we also moved to the next step in our testing process, which was to use a simple methodology to start all of the tests in sync, on all of the virtual machines at the same time. We did this so that we can test the absolute worst case - when all of the virtual machines are overloading the storage system with $\mathrm{I} / \mathrm{O}$ requests. There was one more reason - experience teaches us that we can't use all of the available bandwidth by using just a single object accessing storage to extract the absolute maximum that our storage device can provide.

For this purpose, we used mobaXterm's MultiExec mode to do multi-VM testing and had to overcome a few issues along the way. Specifically, as some of the tests are simple to use within our testing context - we can just start them to run for a specific amount of time. $d d$ utility, on the other hand, doesn't do its job that way - it runs until the test is done, which means that it will run for a variable amount of time on multiple virtual machines. This will be further compounded by the fact that we are running that test on multiple virtual machines at the same time, which will add more latency to the process (summary of latencies plus a bit more random latency), and all of that will have direct negative implications on bandwidth. If we wanted to avoid that, there are VMware-based QoS (Quality of Service) methodologies to do that - via the usage of Storage I/O Control and by creating Virtual Machine Storage Policies. This would be a logical thing to do at the end of our process when we find out the actual performance of our storage device. This is where implementing a semaphore-like functionality and sleep logic (for synchronization) in our script was hugely useful for us as we can sync all of the tests to start at the same time by using it. We included a link to our script in the " Testing methodology and storage performance measurement complexity" section below. 


\section{Testing methodology and storage performance measurement complexity}

We believe that our script clearly shows how involved the programming work behind the virtual machine and storage performance testing premise can be. Our script is available at https:/github.com/vEddYcro/BASH/test-dd.sh, feel free to download it, work with it, modify it, comment on it as it's too long to be directly copy-pasted in this paper. When we dissect our script, there's a clear thought process behind it. Let's explain in detail our design criteria behind the script:

- All local temporary (temp) files are removed from each machine because this is the default place to store the temporary results of any previous tests.

- A DD write test with 64K disk block size (disk mapped to /64k-storage) is performed and for each machine, the final result is written to share (/fiologs/log) in the form $\$$ host-dd-64k-write-final.txt

- DD read test with $64 \mathrm{~K}$ disk block size (disk mapped to /64k-storage) is performed and for each machine, the final result is written to share (/fiologs/log) in the form $\$$ host-dd-64k-read-final.txt

- A DD write test with $8 \mathrm{~K}$ disk block size (disk mapped to /8k-storage) is performed and for each machine, the final result is written to share (/fiologs/log) in the form $\$$ host-dd-8k-write-final.txt

- A DD read test with $8 \mathrm{~K}$ disk block size (disk mapped to /8k-storage) is performed and for each machine, the final result is written to share (/fiologs/log) in the form $\$$ host-dd-8k-read-final.txt

- FIO read test with $8 \mathrm{~K}$ block size is performed on storage with $8 \mathrm{~K}$ block size and final results are written to share (/fiologs/log) in the form \$ host-read-bw-8-8.txt for bandwidth and \$ host-read -IOPS-8-8.txt for IOPS results

- FIO read test with $8 \mathrm{~K}$ block size on storage with $64 \mathrm{~K}$ block size is performed and the final results are written to share (/fiologs/log) in the form \$ host-read-bw-8-64.txt for bandwidth and \$ host-read -IOPS-8-64.txt for IOPS results

- FIO read test with $64 \mathrm{~K}$ block size in storage with $8 \mathrm{~K}$ block size is performed and the final results are written to share (/fiologs/log) in the form \$ host-read-bw-64-8.txt for bandwidth and \$ host-read -IOPS-64-8.txt for IOPS results

- FIO read test with $64 \mathrm{~K}$ block size is performed on storage with $64 \mathrm{~K}$ block size and final results are written to share (/fiologs/log) in the form \$ host-read-bw-64-64.txt for bandwidth and \$ host-read -IOPS-64-64.txt for IOPS results

- FIO test of writing with $8 \mathrm{~K}$ block size on storage with $8 \mathrm{~K}$ block size is performed and the final results are written to share (/fiologs/log) in the form \$ host-write-bw-8-8.txt for bandwidth and \$ host-write -IOPS-8-8.txt for IOPS results

- FIO test of writing with $8 \mathrm{~K}$ block size on storage with $64 \mathrm{~K}$ block size is performed and the final results are written to share (/fiologs/log) in the form \$ host-write-bw-8-64.txt for bandwidth and \$ host-write -IOPS-8-64.txt for IOPS results

- FIO $64 \mathrm{~K}$ block size write test is performed on $8 \mathrm{~K}$ block size storage and final results are written to share (/fiologs/log) in the form \$ host-write-bw-64-8.txt for bandwidth and \$ host-write -IOPS-64-8.txt for IOPS results

- A FIO $64 \mathrm{~K}$ block size write test is performed on a $64 \mathrm{~K}$ block size storage and the final results are written to share (/fiologs/log) in the form \$ host-write-bw-64-64.txt for bandwidth and \$ host-write -IOPS-64-64.txt for IOPS results

- Direct (non-cached) latency check is performed with IOPING tool on $8 \mathrm{~K}$ storage and the result is written to share in the form \$ host-IOPING-DIRECT-8K.txt

- Cached latency check with IOPING tool on $8 \mathrm{~K}$ storage is performed and the result is written to share in the form $\$$ host-IOPING-CACHED-8K.txt

- Direct (non-cached) latency check is performed with the IOPING tool on 64K storage and the result is written to share in the form \$ host-IOPING-DIRECT-64K.txt

- Cached latency check with IOPING tool on $64 \mathrm{~K}$ storage is performed and the result is written to share in the form $\$$ host-IOPING-CACHED-64K.txt

- Summarize the results obtained for all virtual machines into a single result for all variations of tests and save to share (/fiologs/log/TOTAL)

The script also implements a semaphore system that ensures that the next test step does not move until all virtual machines report that they have completed the current test.

\section{Improving storage performance}

By using our script, we got a wealth of data about storage performance. The next step in our optimization process is to use that data to modify hypervisor and storage settings to try to improve virtual machine storage performance. Therefore, this initial dataset can be used as a storage performance baseline that we are going to use to check new data against after we implement some configuration changes to our environment. As with any other technology used for any purpose, there is always a way to optimize the delivery process of a given service, which is true especially in the case of storage systems. Starting from the lowest layer - the hardware used to various software optimizations, the user can almost always expect at least some positive changes in terms of the resulting system performance.

\subsection{Improving storage performance on hardware level}

As various technologies have been used for this paper, it is important to be familiar with how all of them work to find the optimal method of using all of them together in all variants of use. 
Specifically, in this case - the already mentioned optimizations at the lowest layer would be the active use of both server power supplies to ensure that the speed of the PCIe bus is not limited and thus create a "bottleneck" in the data flow at the lowest layer. A lot of servers will drop their PCI Express performance if we have only one power supply installed. In addition, detailed knowledge of the equipment used is crucial, so it is extremely important to get acquainted with its operation and the best way is to study this is the manufacturer's documentation in detail. An example of this would be something seemingly banal like the arrangement of memory modules in the slots on the server - if the memory modules are not set according to the default method defined by the manufacturer it will not be possible to use all the capabilities in our case on a storage server using 2 processors is to fill the memory slots on the server motherboard in a specific order, because otherwise, you can not activate Advanced ECC (Error Correction Code) memory profiles that ensure optimal performance - effectively working memory runs at a lower clock speed and with less bandwidth and thus directly affects the performance of the storage server. Also, separating PCI Express devices across CPUs by using multiple PCI Express riser cards is absolutely crucial if we want to achieve maximum performance from the hardware level.

\subsection{Improving storage performance on the storage network level}

As both FC and Ethernet connections were used for performance metrics testing, it is important to distinguish how both technologies connect to the storage server itself. FC is more of a native plug and play variant when using a direct connection between servers does not require additional configuration nor is there room for additional optimizations on that layer. If multiple hosts were used, i.e. if there was an FC switch between the servers, there would be additional optimization possibilities in terms of the smart definition of FC zones and careful planning of connection methods to maximally mitigate negative scenarios in case of unwanted outages. An example of this would be that, for example, when using multiple FC cards and switches, we don't connect individual FC connectors on a single card to one switch, but that a certain number of connectors of one FC card is connected to one FC switch and thus to another set of connectors of other FC cards and switches. This ensures the maximum available bandwidth in scenarios when a single FC port fails, the card itself, or the switch during which time high availability is constantly ensured in the background. Although performance degradation will occur in this scenario (because some of the uplinks are no longer physically connected) storage service will not be interrupted.

When using Ethernet - iSCSI connections - there are a several settings that can be adjusted to optimize performance primarily because iSCSI as a protocol relies on Ethernet which is relatively universal and its primary purpose is not, as in the case of FC, to provide super-fast, super low-latency method communications with the storage server. These ideas came much later when Ethernet standards were further developed and concepts like RDMA/ROCE were introduced.

Primarily, it is recommended to use network cards that are declared as hardware HBA (Host Bus Adapter), i.e. those that can configure iSCSI initiators in firmware to establish a connection to the server (iSCSI target) before booting the operating system - this way all the work on target-initiator relationship takes place on such a card. That means that no server resources are used and thus better performance is achieved. In our case, the software HBA (iSCSI initiator) was used because such a scenario covers a much higher percentage of usage in real conditions because most companies use this model. If the performance of your hardware bus adapters (HBAs) is unsatisfactory or your SAN storage processors are overutilized, you can adjust your ESXi hosts' maximum queue depth value [4]. Hardware HBA cards are significantly more expensive, so they are often not the first choice. That being said, iSCSI connection is then created via software HBA and the difference in the price is often invested in the storage capacity itself.

Furthermore, in VMware systems, the default queue depth for iSCSI connections is 128 - as this is not a fixed variable (variable name iscsivmk_LunQDepth), it was changed to 256 to make the most of the available storage system. As it is not possible to make a change from the ESXi GUI itself, but only through the terminal by issuing the following command and restarting the system:

esxcli system module parameters set - m iscsi_vmk -p iscsivmk_LunQDepth $=256$

The following command can be used to check if a new value has changed:

esxcli system module parameters list - m iscsi_vmk

In the advanced iSCSI settings on the initiator side (ESXi server) additional things can be set such as:

- Size of each data segment sent via iSCSI (default 128 bytes)

- Parallelism of iSCSI process - the default value is 1 , but as we have a total of 4 used uplinks, this value was set to 4 .

The maximum value can be set to 8 because VMware's hypervisor supports a maximum of 8 iSCSI initiators.

Then, we can move to additional multipathing settings, as this is also something that can be easily changed on VMware's hypervisor. Specifically, we need to configure a Path Selection Policy called Round Robin. Round Robin policy provides load balancing by cycling I/O requests through all Active paths, sending a fixed (but configurable) number of $\mathrm{I} / \mathrm{O}$ requests through each one in turn [5]. 
That means that hypervisor is going to use all available paths to access a block device on our storage system that we exposed. Then, it's about deciding how often will the path between hypervisor and storage change on the I/O or bytes level [6]. For iSCSI devices, it's good to start with IOPS close to 1, while for FC devices, it's best to start around 1000 or 1500. That will depend on the storage device used, but to know more about those details about a specific storage device, we'd need to check the manufacturer's documentation as it's different from manufacturer to manufacturer. For us to be able to configure these settings, we need to know what storage device ID is on the hypervisor level, which is usually its EUI or NAA ID. Then, we would do something similar to this procedure for iSCSI block device with EUI ID eui.3435353637303632 - to set both PSP and IOPS settings:

esxcli storage nmp device set --device eui.3435353637303632 --psp VMW_PSP_RR

esxcli storage nmp psp roundrobin deviceconfig set --type=iops --iops $=1$--device $=$ eui.3435353637303632

esxcli storage core claimrule load

esxcli storage nmp device list

Also, we'd do something like this on FC block device with EUI ID eui.3438316432326162, to set both PSP and IOPS settings:

esxcli storage nmp device set --device eui.3438316432326162 --psp VMW_PSP_RR

esxcli storage nmp psp roundrobin deviceconfig set --type $=$ iops - -iops $=1500--\bar{d}$ evice $=$ eui.3438316432326162

esxcli storage core claimrule load

esxcli storage nmp device list

We used these two EUI IDs as these were the ones generated by the storage system, and these IDs will vary per environment.

The final thing we can optimize on the network layer for iSCSI is the MTU (Maximum Transmission Unit). The default value is 1500, but support for jumbo frames can be turned on and the MTU value can be effectively set to 9000 . This enables the transmission packets that are six times the size on the network layer and thus reduces losses that would be caused by encapsulation of the packet itself. thus less usable bandwidth is lost to traffic encapsulation, which translates to overhead and latency. To achieve this, it is important that all devices (physical and virtual) must be set to use an MTU with a value of 9000 . This refers to:

- VMkernel port (ESXi)

- vSwitch (ESXi)

- physical switch ports

- any network card that serves as an iSCSI target (OSNEXUS)

Some of these changes aren't trivial and require a bit of time and effort. But in the end, we are usually going to be rewarded with much better performance.

\subsection{Improving storage performance on the storage level}

Regardless of the connection medium used (FC or Ethernet), certain things can also be optimized on the storage server itself, such as default profiles for individual disk volumes. The default IO profile in OSNEXUS is "Virtualization", which is adequate for most uses in providing storage systems to any type of hypervisor (Hyper-V, ESXi,...), but when we check the exact specifications of this profile, we see that in this profile max queue depth variable is set to 128. Since we use a queue depth of 256 in our tests to make the most of the available potential of the IOPS value (which would be desirable for data storage systems for databases, etc.), this value must be changed.

Reviewing other available profiles, we find that the profile "SSD (Default)" uses the default value of max queue depth of 256 and that other variables of this profile mostly reflect our scenario, so this profile was selected for all tests. In case there is no already predefined profile that would suit us, you can always create a new one, with parameters specified by user input.

\section{Conclusion}

We proposed a two-step, iterative process of measuring storage performance. First, we set up our environment, start our script and let it complete its run, and use acquired data as a baseline. Then, we start introducing changes to environment configuration so that we can extract more storage performance out of our storage system and iterate over this step.

There are two harsh realities to be aware of here: 
- This procedure will take time and it is a bit complicated, but it will give us much, much more performance. By using this methodology, we've seen storage bandwidth increase from 700-800 MB/s to 2-2.5 GB/s. We've also noticed significant increases in terms of IOPS, but not as much. Bandwidth and IOPS are often in the inversely proportional relationship, depending on the workload.

- OSNEXUS, as a product, is cheap, and extracting these levels of performance from it is stunning. Comparing our storage solution in terms of pricing and performance vs some kind of commercial storage device capable of the same numbers is something that usually puts commercial storage devices to shame, as they're either slower, or much more expensive. We can confirm this from the past five years of experience working with it.

Implementing these changes had a significant impact on how our storage device performed - performance was significantly better. There's a bit of trial and error involved here to find the correct combination, but it usually doesn't take a lot of time. Also, it enables us to have quite a bit of freedom to tune our storage performance towards more bandwidth (MB/s or GB/s), IOPS, or both. That means that we can create multiple block devices, tune them differently for different purposes, which is a rather cheap way of having application-specific storage devices, tuned for specific applications. At the end of the day, this is where the storage industry has been going for the past couple of years. And, to top it off, the raw performance is excellent, and on par with commercial storage systems 10x the price, or more.

Next research steps could include making all of this completely automated and orchestrated by using Ansible to do everything from copying our script to virtual machines to starting tests. Next, we could also make modifications to our script to use SSH keys to automatically connect to all VMs and be completely automated, without having to use mobaXterm's MultiExec mode. After that, we could chase even more performance out of our storage system by implementing the same system in containers and use Docker or Podman to spool up containers for testing instead of virtual machines. There are reasons why containerization has been a key cloud computing platform that can perfectly take advantage of this parallelism by allowing multiple isolated instances (i.e., containers) of the storage resources [8]. That would mean significantly less overhead as containers have less compute and storage overhead than virtual machines. If we wanted to, we could also implement all of the storage tests as functions, and then create some kind of algorithm to randomize these tests so that different tests are started at the same time to create a completely random scenario. So, there are plenty of opportunities for further research and development of the principle.

\section{References}

[1] Dakic, V. (2019). Influence of virtual storage controller type on Microsoft SQL Server 2019 performance, Proceedings of DAAAM., at Zadar, Croatia, ISSN 1726-9679, doi:10.2507/30th.daaam.proceedings.050

[2] Liu, J.; Wang, F.; Feng, D.; (2019). CostPI: Cost-Effective Performance Isolation for Shared NVMe SSDs, ICPP 2019, Kyoto, Japan, doi:10.1145/3337821.3337879

[3] Dennerman, F.; Hagoort, N. (2017). VMware vSphere 6.5 Host Resources Deep Dive, Frank Dennerman and Niels Hagoort, ISBN 9781540873064

[4] Elder, K.; Kusek, Cristopher; Sarkar, P. (2017). vSphere High Performance Cookbook, Packt Publishing, ISBN: 978-1-78646-462-0

[5] VMware (2015). Performance Best Practices for VMware vSphere 6.0, VMware

[6] VMware (2021.) Adjusting Round Robin IOPS limit from default 1000 to 1 (2069356), URL: https://kb.vmware.com/s/article/2069356

[7] Gastermann, B.; Stopper, M.; Kossik A.; Katalinic, B. (2014). Secure Implementation of an On-Premises Cloud Storage Service for Small and Medium-Sized Enterprises, 25th DAAAM International Symposium on Intelligent Manufacturing and Automation, Procedia Engineering 100, doi: 10.1016/j.proeng.2015.01.407

[8] Yang, Z.; Hoseinzadeh, M.; Wong, P.; Artoux, J.; Mayers, C.; Evans, D.T.; Bolt, R.T.; Bhimani, J.; Mi, N.; Swanson, S. (2017). H-NVMe: A Hybrid Framework of NVMe-based Storage System in Cloud Computing Environment, 36th International Performance Computing and Communications Conference (IPCCC), ISSN: 2374-9628, DOI: 10.1109/PCCC.2017.8280431. 